\title{
Recurrent Malignant Skin Neoplasm
}

National Cancer Institute

\section{Source}

National Cancer Institute. Recurrent Malignant Skin Neoplasm. NCI Thesaurus. Code C150545.

Reemergence of a malignant skin neoplasm after a period of remission. 